CERN-TH-7044/93

ENSLAPP-A-441/93

\title{
Axion Emission from Red Giants and White Dwarfs
}

\author{
T. Altherr巾 \\ Theory Division, CERN, CH-1211 Geneva 23, Switzerland \\ E. Petitgirard \\ Laboratoire de Physique Théorique ENSLAPP \& \\ B.P. 110, F-74941 Annecy-le-Vieux Cedex, France \\ and \\ T. del Río Gaztelurrutia \\ Departamento de Física Aplicada \\ Escuela de Ingenieros, Alameda de Urquijo $S / N$ \\ S-48013 Bilbao, Spain
}

\begin{abstract}
Using thermal field theory methods, we recalculate axion emission from dense plasmas. We study in particular the Primakoff and the bremsstrahlung processes. The Primakoff rate is significantly suppressed at high densities, when the electrons become relativistic. However, the bound on the axion-photon coupling, $G<10^{-10} \mathrm{GeV}$, is unaffected, as it is constrained by the evolution of HB stars, which have low densities. In contradistinction, the same relativistic effects enhance the bremsstrahlung processes. From the red giants and white dwarfs evolution, we obtain a conservative bound on the axion-electron coupling, $g_{a e}<2 \times 10^{-13}$.
\end{abstract}

CERN-TH-7044/93

October 93

\footnotetext{
${ }^{1}$ On leave of absence from L.A.P.P., BP110, F-74941 Annecy-le-Vieux Cedex, France

${ }^{2}$ URA 14-36 du CNRS, associée à l'E.N.S. de Lyon, et au L.A.P.P. d'Annecy-le-Vieux.
} 


\section{Introduction}

There are recent speculations that axions [1] might play a role in astrophysics [2, 3]. Indeed, the cooling rate of the white dwarf G117-B15A has recently been measured and is faster than expected from any conventional model. Axions with masses of the order of $8( \pm 3) \times 10^{-3} / \cos ^{2} \beta \mathrm{eV}$ would provide an additional cooling mechanism and reconcile models with the data [2].

On the other hand, it is well known that other constraints on axions come from astrophysical systems such as red giants and SN1987A [4]. A rather well-established bound comes from the evolution of red giants [5]. The upper bound for DFSZ axions is $m_{a}<1.1 \times 10^{-2} / \cos ^{2} \beta \mathrm{eV}$, quite close to the proposed solution for the cooling mechanism of G117-B15A.

The calculations of the axion emission rates in stellar cores involve a quite complicated physics (screening and correlation effects). They are based upon kinetic theory [6]. As a consequence of this complexity, there has been a considerable amount of confusion in identifying the correct physics for the photon propagation in dense media [7, 8]. The best approach is due to Raffelt [5, 9], and we shall reproduce some of his results here. Indeed, given the outstanding implications of the possible existence of axions, we propose to recalculate the dominant processes of axion emission in red giants and white dwarfs. We shall use a different method, derived from thermal field theory [10, 11. The advantage of this method is that it already includes the classical limit, but allows also systematic diagrammatic studies of the higher-order effects [12].

The paper is organized as follows: in Sect. 2 we recall some of the basic ingredients concerning the spectral density of photons at finite temperature and density. In Sect. 3, we investigate the Primakoff process in details. We confirm the previous bound on the axion-photon coupling strength. In Sect. 4, we calculate the axion emission from electron-nucleus bremsstrahlung through the exchange of transverse and longitudinal photons. We discuss our results in Sect. 5. From the red giants and white dwarfs evolution, we obtain a conservative bound on the axion-electron coupling, $g_{a e}<2 \times 10^{-13}$. 


\section{The method}

The general framework and methods of gauge field theory at finite temperature and density have been discussed extensively elsewhere [12], and we shall not repeat them here. Let us just recall that in the plasma core of dense stars, matter effects are properly taken into account by the finite temperature and density corrections to the polarization tensor $\Pi(Q)$. In a heat bath, there exist two modes of propagation of electromagnetic waves, transverse (T) and longitudinal (L). In calculating an electromagnetic scattering, it is particularly convenient to make use of the spectral density [13]

$$
\begin{aligned}
\rho_{\mathrm{T}, \mathrm{L}}(Q) & =\frac{1}{\pi} \operatorname{Re} \frac{i}{Q^{2}-\Pi_{\mathrm{T}, \mathrm{L}}(Q)+i \epsilon} \\
& =\delta\left(Q^{2}-\operatorname{Re}_{\mathrm{T}, \mathrm{L}}(Q)\right)-\beta_{\mathrm{T}, \mathrm{L}}(Q) .
\end{aligned}
$$

Our convention is to use capital letters only for quadri-momenta, e.g. $Q=(\omega, \mathbf{q})$. Depending on whether the photon is time-like or space-like, the above quantity describes two different modes:

i) If the photon is time-like $\left(Q^{2}>0\right)$, the spectral density turns to a $\delta$-function and describes a quasi-particle mode (plasmon). This quasi-particle obeys a dispersion relation given, in the non-relativistic limit, by $\omega^{2}=\omega_{0}^{2}+q^{2}$ for the transverse mode, and by $\omega^{2}=\omega_{0}^{2}$ for the longitudinal one. Similar, although much more involved, analytic expressions can be derived in the ultra-relativistic limit. Recent works have shown that it is possible to avoid the non/ultra-relativistic limit and get closed formulae [14, 15]. The plasmon frequency is given by $\omega_{0}^{2}=e^{2} N_{e} / m_{e}$ for non-degenerate and non-relativistic plasmas and by $\omega_{0}^{2}=e^{2} N_{e} / \mu$ for degenerate plasmas ( $\mu$ is the relativistic electron chemical potential, $\mu=\sqrt{p_{F}^{2}+m_{e}^{2}}$. In the non-relativistic case, the longitudinal dispersion relation crosses the light-cone at $q=\omega_{0}$, and eventually continues for large values of $q$ until an imaginary part develops in $\Pi_{\mathrm{L}}(Q)$. This peculiar feature of a space-like quasi-particle occurs only in a medium. It will be particularly relevant for the Primakoff effect, as we shall see in the next section.

ii) At leading order, the polarization tensor develops an imaginary part only below the light-cone, for instance when $\omega<p_{F} q / \mu$ in the degenerate case. In the hard-loop approximation, the imaginary parts of the transverse and longitudinal components 
are given by

$$
\begin{aligned}
& \operatorname{Im}_{\mathrm{T}}(Q)=\alpha \frac{1}{q} \int_{m}^{\infty} d E\left(\frac{\omega^{2}}{q^{2}} E^{2}-p^{2}\right) \theta(p q-\omega E)\left[n_{F}\left(E-\frac{\omega}{2}\right)-n_{F}\left(E+\frac{\omega}{2}\right)\right] \\
& \operatorname{Im}_{\mathrm{L}}(Q)=2 \alpha\left(1-\frac{\omega^{2}}{q^{2}}\right) \frac{1}{q} \int_{m}^{\infty} d E E^{2} \theta(p q-\omega E)\left[n_{F}\left(E-\frac{\omega}{2}\right)-n_{F}\left(E+\frac{\omega}{2}\right)(2,)\right.
\end{aligned}
$$

where the contribution from antiparticles has been dropped. The spectral density then turns to some kind of Lorentzian with a complicated structure. One may notice that longitudinal and transverse expressions are very similar in the ultra-relativistic limit [12], but not in the non-relativistic case [14]. In the non-relativistic and nondegenerate case, we recover the standard relation [6]

$$
\operatorname{Im} \Pi_{\mathrm{L}}(Q)=\sqrt{\pi} k_{D}^{2} z e^{-z^{2}} \quad \text { with } \quad z=\frac{\omega}{q} \sqrt{\frac{m}{2 T}},
$$

and $k_{D}$ is the Debye mass corresponding to the screening of static electric fields. In non-degenerate plasmas, $k_{D}^{2}=e^{2} N_{e} / T$, and in degenerate plasmas, $k_{D}^{2}=\left(e^{2} / \pi^{2}\right) \mu p_{F}$.

As we shall see in the following, it is important to notice that screening effects are absent for transverse modes, while there appears a mass term into the denominator of the longitudinal spectral density. More precisely, one has in the static limit

$$
\begin{aligned}
\operatorname{Re}_{\mathrm{T}}(\omega \rightarrow 0, q) & =k_{D}^{2} \frac{\omega^{2}}{q^{2}} \\
\text { and } \operatorname{Re}_{\mathrm{L}}(\omega \rightarrow 0, q) & =k_{D}^{2} .
\end{aligned}
$$

Notice that $k_{D}$ also appears in the expression for the transverse propagator. Because of the $\omega$ dependence, one may call this effect a dynamical screening [13].

All the above equations have been calculated for the case of electrons or protons. Similar expressions can be derived in case of ions, using scalar electrodynamics instead of quantum electrodynamics. This is legitimate as, in most scatterings, the photon does not see the inner structure of the nucleus. Moreover, one does not need form factors as we only consider the thermal corrections, which are ultravioletfinite. Equations (3) and (4) are identical, except for the Debye mass, which becomes $k_{D}^{2}=Z^{2} e^{2} N_{\text {ions }} / T$.

The scattering rates are computed either using the cutting rules of Kobes and Semenoff [16] in the real-time formalism [11], or by analytic continuation in the imaginary-time formalism [17]. Many examples can be found in the literature, including those discussed here, but mostly in the ultra-relativistic regime [12, 15, 18, 19]. 


\section{The Primakoff effect}

In a previous work [12], the axion emission through photon exchange was already studied in detail for the case of an ultra-relativistic plasma. This analysis can be extended to the general case of relativistic as well as non-degenerate plasmas. As shown in [12], a systematic study of higher-order effects involves a resummation of a class of diagrams. This resummation is best implemented within Thermal Field Theory. In this approach, the axion emission rate is calculated through the discontinuity of its self-energy. This procedure avoids the complication of using Feynman scattering amplitudes, and yields the contribution of several different processes to the axion production. Nevertheless, it remains difficult to compare the results thus obtained with kinetic theory calculations [5].

When we calculate the discontinuity of the axion self energy, three different kinds of contributions naturally appear. Out of them, the "pole-cut" term is what we can best compare with the classical Primakoff process. It involves the scattering of a time-like plasmon with a target (which can either be an ion or an electron) through the exchange of a space-like plasmon. To be able to compare our results with previous calculations, we shall concentrate on the case where the time-like photon is transverse, while the space-like one is longitudinal. The equivalence between Primakoff and polecut is exact when the photon energies are hard, i.e. larger than the Debye mass or the plasmon frequency; it ceases to be valid when the photons are soft, as the space-like photon is affected by Landau damping.

In our calculation of the axion emission of stars, we shall first compute the dominant pole-cut contribution, then discuss when the pole-pole contribution becomes relevant, and finally find a complete expression valid in the non-degenerate, nonrelativistic case.

Following previous work [12], we start from the expression for the pole-cut contribution to the emission rate of axions

$$
\Gamma_{P C}(K)=\frac{G^{2}}{8 \pi k} \int_{\omega_{0}}^{\infty} d \omega \int_{-1}^{+1} \sin \theta d \theta q n_{B}(\omega)\left(1+n_{B}\left(\omega^{\prime}\right)\right) Z_{2}(Q) \beta\left(Q^{\prime}\right) g\left(Q, Q^{\prime}\right)
$$

where the same notation as in [12] is used $\left(Q=Q^{\prime}+K\right)$. In the last expression, $Q^{\prime}$ is the momentum of the space-like, longitudinal plasmon, while $Q$ is the momentum 
of the transverse, on-shell photon (see Fig. 1). For a pseudoscalar coupling one has $g\left(Q, Q^{\prime}\right)=q^{2} Q^{\prime 2} \sin ^{2} \phi$, where $\phi$ is the angle between $Q$ and $Q^{\prime}[18$.

The non-relativistic dispersion relations for a transverse photon give

$$
\omega^{2}=q^{2}+\omega_{0}^{2} \quad \text { and } \quad Z_{2}(Q)=1
$$

In fact, the dispersion relation for the transverse photon in the non-relativistic limit differs only very slightly from the ultrarelativistic one [14, 15]. We assume that, in the calculation of the pole-cut term, the approximation of the dispersion relation given above can be used in all regimes to a good accuracy. We need also the 'cut' contribution of the spectral density of the longitudinal plasmon. As a first approximation we shall take

$$
\beta_{\mathrm{L}}\left(Q^{\prime}\right) \simeq-\frac{1}{\pi} \frac{\operatorname{Im} \Pi_{\mathrm{L}}\left(Q^{\prime}\right)}{\left(q^{\prime 2}+k_{D}^{2}\right)^{2}}
$$

where the longitudinal plasmon is static. As the imaginary part of $\Pi_{\mathrm{L}}$ only exists for $\omega^{\prime}<v_{*} q^{\prime}$, we expect this approximation to hold well in the case of a non-relativistic plasma, where the ion velocity $v_{*}$ is indeed very small, both in a degenerate and in a non-degenerate electron gas. The Debye mass appearing in the photon propagator is in fact the sum of the different contributions to the screening (electrons, protons and dominantly ions contributions).

The static limit allows a direct evaluation of

$$
\int d \omega^{\prime}\left(1+n_{B}\left(\omega^{\prime}\right)\right) \operatorname{Im} \Pi_{\mathrm{L}} \simeq T \int \frac{d \omega^{\prime}}{\omega^{\prime}} \operatorname{Im} \Pi_{\mathrm{L}} \simeq \pi T k_{D}^{2}
$$

After performing the remaining integration on $\theta$, one gets the final result for the pole-cut contribution to the emission rate of axions

$$
\Gamma(K)=\frac{G^{2}}{8 \pi} T k_{D}^{2} n_{B}(k) \sqrt{1-\frac{\omega_{0}^{2}}{k^{2}}}\left[\frac{2 k^{2}-\omega_{0}^{2}+k_{D}^{2}}{4 k \sqrt{k^{2}-\omega_{0}^{2}}} \ln \frac{\left(\sqrt{k^{2}-\omega_{0}^{2}}+k\right)^{2}+k_{D}^{2}}{\left(\sqrt{k^{2}-\omega_{0}^{2}}-k\right)^{2}+k_{D}^{2}}-1\right] .
$$

When the axion momentum $k$ is much larger than the plasmon frequency $\omega_{0}$, the expression in square brackets simplifies to

$$
[\ldots] \rightarrow\left[\frac{1}{2}\left(1+\frac{k_{D}^{2}}{2 k^{2}}\right) \ln \left(1+\frac{4 k^{2}}{k_{D}^{2}}\right)-1\right]
$$

The main approximation in the calculation of the pole-cut contribution is the static limit for the longitudinal space-like plasmon. This approximation should work well 
in a large variety of regimes, as it is the interaction with the ions that contributes most to both the Debye length and the imaginary part of $\Pi_{\mathrm{L}}$. The ions being always non-relativistic, our approximation is well justified.

The pole-pole contribution corresponds to the decay of a transverse photon into an axion and a plasmon. When calculating it, we must use the longitudinal plasmon dispersion relation. In the low-momentum limit, this dispersion relation gives a constant energy $\omega_{0}$, while in the large momentum limit, it tends asymptotically to $\omega=v_{*} q$. Moreover, the Jacobian includes a factor $\omega-v_{*} q$, which suppresses the process once the asymptote is reached. In the case of a very dense plasma, the asymptote coincides with the light cone, and only $q$ 's of the order of or smaller than the frequency of the plasma contribute. Nevertheless, the phase space increases considerably in the case of non-relativistic, dilute plasmas, where $q$ can be as large as $\omega / v_{*}$. Notice that in this case we have a space-like solution for the longitudinal particle-like propagation in the plasma.

It is hard to find a good analytical approximation for the pole-pole contribution in regions other than non-relativistic and non-degenerate. Therefore, we perform the calculation numerically. We start again from the results of 12,

$$
\Gamma_{P P}(K)=\frac{G^{2}}{8 \pi k} \int_{\omega_{0}}^{\infty} d \omega n_{B}(\omega)\left(1+n_{B}\left(\omega^{\prime}\right)\right) Z_{2}\left(Q^{\prime}\right) Z_{2}(Q) g\left(Q, Q^{\prime}\right) .
$$

Here, $\omega(q)$ and $Z_{2}(Q)$ are the same as in Eq. (6), while the longitudinal dispersion relations give 14

$$
q^{\prime 2}=\frac{3 \omega_{0}^{2}}{v_{F}^{2}}\left[1-\frac{1}{2} \frac{\omega^{\prime}}{v_{F} q^{\prime}} \ln \frac{\omega^{\prime}+v_{F} q^{\prime}}{\omega^{\prime}-v_{F} q^{\prime}}\right] \quad \text { and } \quad Z_{2}\left(Q^{\prime}\right)=\frac{2}{3} \frac{q^{\prime 2}}{Q^{\prime 2}}\left(\frac{\omega^{\prime 2}-v_{F}^{2} q^{\prime 2}}{\omega_{0}^{2}-\omega^{\prime 2}+v_{F}^{2} q^{\prime 2}}\right) .
$$

We show the result of our numerical calculation in Fig. 2. The pole-pole contribution gets large at low densities, and is indeed dominated by the exchange of space-like longitudinal plasmons. The curve follows remarkably the pole-cut term, that is Eq. (9). At high densities, though, the pole-pole is dominated by the exchange of time-like longitudinal plasmons, and is still $\sim 50 \%$ of the pole-cut rate. However, in this region, the rate is dominated by the pole-cut term with exchange of a space-like transverse plasmons (we shall comment more in detail on that point below). 
We have also plotted the production rate predicted by Raffelt. We see that our prediction only agrees in the region of low densities. We shall argue that this is consistent with the approximations used in both calculations.

In his calculation, Raffelt used a form factor different from the standard DebyeHückel one. His justification of this point relies in the stationarity of the targets. The relevant time scale for a Primakoff-type event to occur is the time it takes a photon to cross a region of the size of the Debye length. If the incident photon is very energetic this time will be $k_{D}^{-1}$, much smaller than $v_{e} k_{D}^{-1}$, the time an electron takes to cross the same region. But whenever the temperature is of the order of $\omega_{0}$, the velocity of the average plasmon excitation will be much smaller than the speed of light, and the argument above will cease to be correct. Indeed, we see from our calculation that when the densities grow, the pole-cut term becomes dominant. In fact, it is easily proved that our pole-cut contribution is equivalent to the result that would be obtained using the standard Debye-Hückel form factor in the calculations of [5].

Within the non-relativistic, non-degenerate approximation, a compact result for the axion emission rate can be deduced, which reproduces exactly the result of ref. [5]. If we consider both pole-pole and pole-cut contributions together, the axion emission rate becomes

$$
\Gamma(K)=\frac{G^{2}}{8 \pi k} \int_{\omega_{0}}^{\infty} d \omega \int_{-1}^{+1} \sin \theta d \theta q n_{B}(\omega)\left(1+n_{B}\left(\omega^{\prime}\right)\right) Z_{2}(Q) \rho_{\mathrm{L}}\left(Q^{\prime}\right) g\left(Q, Q^{\prime}\right),
$$

where $\rho_{\mathrm{L}}$ is now the complete spectral density for the longitudinal plasmon. In terms of the electric permittivity $\epsilon_{L}$, one has [6]

$$
\beta_{\mathrm{L}}\left(Q^{\prime}\right)=-\frac{1}{Q^{\prime 2}} \operatorname{Im} \frac{1}{\epsilon_{\mathrm{L}}}
$$

In the classical limit, Eq. (13) can be approximated to

$$
\Gamma(K)=\frac{G^{2}}{8 \pi} k^{2} n_{B}(k) \int_{-\infty}^{\infty} d \omega^{\prime} \frac{T}{\omega^{\prime}} \int_{-1}^{+1} d(\cos \phi) \sin ^{2} \phi\left(-\operatorname{Im} \frac{1}{\epsilon_{\mathrm{L}}}\right) .
$$

At this stage, it is quite convenient to use the Kramers-Krönig sum rule [6]

$$
\int_{-\infty}^{+\infty} \frac{d \omega^{\prime}}{\omega^{\prime}} \operatorname{Im} \frac{1}{\epsilon_{\mathrm{L}}}=-\pi \frac{k_{D}^{2}}{q^{\prime 2}+k_{D}^{2}}
$$


We are left with the angular integration, which can be solved exactly, leading to the final result:

$$
\Gamma(K)=\frac{G^{2}}{16 \pi} T k_{D}^{2} n_{B}(k)\left[\left(1+\frac{k_{D}^{2}}{4 k^{2}}\right) \ln \left(1+\frac{4 k^{2}}{k_{D}^{2}}\right)-1\right],
$$

which indeed coincides with ref. [5]. We notice that in the large- $k$ region, the sum rules are fulfilled by the pole-cut term, while in the low- $k$ region the pole-pole dominates.

Let us now comment on two other points:

i) The main approximation, which leads to our final result, is the static limit in the space-like photon propagator. It is obviously an overestimate. However, it is certainly a very good approximation in the non-relativistic limit as $\omega<q p / E=q \sqrt{3 T / M} \simeq$ $0.003 q$ (for ${ }^{4} \mathrm{He}$ ). As axions mostly originates from ion scatterings, we see that this approximation is well justified.

ii) In Fig. 2, we also show the contribution coming from the exchange of transverse photons. Unfortunately, we have not been able to compute analytically the axion emission rate in this case, except in a few limiting cases. However, we can use the numerical codes developed previously for the ultra-relativistic regime [18, 12]. With the help of the results of [14, 15], the implementation of the general case of a degenerate gas of electrons is straightforward. Notice that, in this case, the dominant process comes from scatterings where the targets are not ions but electrons. Still, the Debye mass that one has to use in the photon propagator (see Eq. (4)) is the sum of all the contributions.

The justification for neglecting transverse space-like excitations is because of their usual small fluctuations in the non-relativistic limit [9, 14]. However, and contrary to the longitudinal case, these excitations are not screened and can therefore contribute significantly when the Debye mass gets large. Also, as the degeneracy of the electron gas increases, the spectral density of transverse excitations becomes as large as the longitudinal ones. And this is definitely the case in red giants, when the density reaches $\rho=10^{6} \mathrm{~g} / \mathrm{cm}^{3}$ [14].

The numerical results shown in Fig. 2 clearly demonstrate this feature. 


\section{Bremsstrahlung}

Another important contribution to the emission rate of axions can be the bremsstrahlung process. In this case, a plasmon is absorbed by an electron or ion, and an axion is emitted. In contradistinction to a Primakoff-type process, bremsstrahlung

occurs via the fermion-axion coupling, $-i g \bar{\Psi} \gamma_{5} \Psi \Phi_{a}$. A derivative axion coupling was introduced by Raffelt, to set right the potentially incorrect consequences of the usual pseudoscalar coupling. Nevertheless, in the case of the bremsstrahlung process, it can easily be shown that both couplings can be used, and lead to identical results.

In the case of ultra-degenerate plasmas of electrons, several approximations can be performed. On the one hand, Pauli-blocking effects imply that only electrons near the Fermi sphere contribute [12]. These electrons are very energetic, with energies of the order of $\mu$, and the hard-loop approximation will be justified. Another consequence of Pauli-blocking is the strong suppression of the $e^{-} e^{-}$bremsstrahlung processes in comparison to $e^{-}$-ion ones.

We shall be concerned with the imaginary part of the polarization tensor, taking only into account the exchange with the nuclei in the photon propagator. We only consider the space-like photon, as the time-like photon is related with the Compton process, which has been shown to be subleading [1]. In a strongly degenerate plasma, this is easy to understand, as the plasmon density is automatically damped by a factor $e^{-\omega_{0} / T}$. Using the cutting rules of Kobes and Semenoff, the emission rate is written as (see Fig. 3)

$$
\begin{aligned}
\Gamma(K)= & \frac{g_{a e}^{2}}{k} \int \frac{d^{4} P}{(2 \pi)^{3}} \frac{d^{4} Q}{(2 \pi)^{3}}\left(\theta\left(p_{0}-k_{0}\right)-n_{F}\left(p_{0}-k_{0}\right)\right) \delta\left((P-K)^{2}-m_{e}^{2}\right) \\
& \times\left(\theta\left(q_{0}-p_{0}\right)-n_{F}\left(p_{0}-q_{0}\right)\right) \delta\left((P-Q)^{2}-m_{e}^{2}\right) \\
& \times\left(\theta\left(-q_{0}\right)+n_{B}\left(q_{0}\right)\right) \beta(Q) \sum_{\text {spins }}|\mathcal{A}|^{2},
\end{aligned}
$$

where the amplitude is given by

$$
\mathcal{A}=\epsilon_{\mu}^{s}(Q) \bar{u}^{r^{\prime}}(P-K)\left[\gamma^{5} \frac{P+m_{e}}{P^{2}-m_{e}^{2}} \gamma^{\mu}+\gamma^{\mu} \frac{P-Q-K+m_{e}}{(P-Q-K)^{2}-m_{e}^{2}} \gamma^{5}\right] u^{r}(P-Q)
$$

After summing over all spins and photon polarizations, we can split the total squared matrix element into its longitudinal and transverse parts. Using the hard-loop ap- 
proximation, we obtain,

$$
\begin{aligned}
\sum_{\text {spins }}|\mathcal{A}|_{\mathrm{T}}^{2} & =\frac{-2}{(P \cdot K)^{2}}\left[2(K \cdot Q)^{2}+Q^{2} k^{2}\left(-1+(\hat{\mathbf{k}} \cdot \hat{\mathbf{q}})^{2}\right)\right] \\
\sum_{\text {spins }}|\mathcal{A}|_{\mathrm{L}}^{2} & =\frac{-2 k^{2} Q^{2}}{(P \cdot K)^{2}}\left[1-(\hat{\mathbf{k}} \cdot \hat{\mathbf{q}})^{2}\right] .
\end{aligned}
$$

In a very dense plasma, Fermi distribution functions can be well approximated by step functions. This approximation yields the following constraints on the energies

$$
\mu+k_{0}<p_{0}<\mu+q_{0}
$$

and allows us to write

$$
n_{B}\left(q_{0}\right) n_{F}\left(p_{0}-q_{0}\right)\left(1-n_{F}\left(p_{0}-k_{0}\right)\right) \simeq n_{B}\left(q_{0}\right)\left(1+n_{B}\left(q_{0}-k_{0}\right)\right)\left(q_{0}-k_{0}\right) \delta\left(p_{0}-\mu\right) .
$$

At this stage, the integrations over $p_{0}$ and $p$ are easily solved, leading to

$$
\begin{aligned}
\Gamma_{T, L}(K)= & \frac{g_{a e}^{2}}{k} p_{F} \int \frac{d^{4} Q}{(2 \pi)^{4}} \int \frac{d \Omega_{p}}{8 \pi^{2}} \beta_{T, L}(Q) n_{B}\left(q_{0}\right)\left(1+n_{B}\left(q_{0}-k_{0}\right)\right) \\
& \times\left(q_{0}-k_{0}\right) \delta\left((P-Q)^{2}-m_{e}^{2}\right) \sum_{\text {spins }}|\mathcal{A}|_{T, L}^{2} .
\end{aligned}
$$

We shall first calculate the transverse contribution to the emission rate, and proceed later to the longitudinal case. Before performing the angular integrations, let us have a look at the spectral densities of the exchanged photons. The exchange with the nuclei involves the space-like contribution of the spectral density (Eq. (1)). In the case of heavy, non-relativistic ions we can use the Boltzman statistical factor in the calculation of $\operatorname{Im} \Pi_{\mathrm{T}}$, and we obtain:

$$
\operatorname{Im} \Pi_{\mathrm{T}}=-\sqrt{\frac{\pi}{6}} k_{D}^{2} v_{*} \frac{T}{q} e^{\frac{-q_{0}}{2 T}} n_{B}^{-1}\left(q_{0}\right) e^{-\frac{q_{0}^{2}}{q^{2}} \frac{3}{2 v_{*}^{2}}},
$$

where $v_{*}^{2}=3 T / M \ll 1$ is the average speed of ions and $M$ is the mass of the nuclei. The exponential factor allows us to approximate $q_{0} \ll q$. Moreover, if we recall that screening effects are absent for transverse modes, we see that $\operatorname{Re} \Pi_{\mathrm{T}} \ll q^{2}$ and $\mathrm{Im}_{\mathrm{T}} \ll q^{2}$. Therefore the transverse spectral density has the following form

$$
\beta_{\mathrm{T}}(Q) \simeq-\frac{1}{\pi} \frac{\operatorname{Im} \Pi_{\mathrm{T}}(Q)}{q^{4}}
$$


which just corresponds to using bare propagators. Performing the angular integrations is not a trivial task, since three combined angles appear, namely $\hat{\mathbf{k}} \cdot \hat{\mathbf{q}}, \hat{\mathbf{k}} \cdot \hat{\mathbf{p}}$ and $\hat{\mathbf{p}} \cdot \hat{\mathbf{q}}$. To ease the calculation, a usual trick, based on the isotropy of the emission rate, can be used,

$$
\Gamma(K)=\frac{1}{4 \pi} \int d \Omega_{k^{\prime}} \Gamma\left(K^{\prime}\right) .
$$

Notice that if $\hat{\mathbf{k}} \cdot \hat{\mathbf{p}}$ for fixed $\mathbf{p}$ is not constrained, $\hat{\mathbf{q}} \cdot \hat{\mathbf{p}}$ for fixed $\mathbf{q}$ is on the other hand bounded.

We arrive at an expression for $\Gamma$ where only one integral is left unsolved,

$$
\begin{aligned}
\Gamma_{\mathrm{T}}(K)= & \frac{g_{a e}^{2}}{192 \pi^{4}} \frac{k_{D}^{2} v_{*}^{2} T}{k p_{F}^{2}}\left[\frac{\mu}{p_{F}} \ln \frac{\mu+p_{F}}{\mu-p_{F}}+2\left(\frac{p_{F}^{2}}{m_{e}^{2}}-1\right)\right] \\
& \times \int_{0}^{\infty} d q_{0}\left(1+n_{B}\left(q_{0}\right)\right) \frac{q_{0}}{q_{0}+k} e^{-\frac{q_{0}}{2 T}} e^{-\frac{k}{2 T}}
\end{aligned}
$$

Finally, we can consider the energy loss rate per volume, related to the emission rate by

$$
Q_{\mathrm{T}}=\frac{1}{2 \pi^{2}} \int_{0}^{\infty} d k k^{3} \Gamma_{\mathrm{T}}(K)
$$

From the last expression of the emission rate, we can deduce the value of the energy loss rate for a transverse photon exchanged,

$$
\begin{aligned}
Q_{\mathrm{T}}= & \frac{g_{a e}^{2}}{48 \sqrt{2} \pi^{6}} \frac{k_{D}^{2} v_{*}^{2} T^{5}}{p_{F}^{2}}\left[\frac{\mu}{p_{F}} \ln \frac{\mu+p_{F}}{\mu-p_{F}}+2\left(\frac{p_{F}^{2}}{m_{e}^{2}}-1\right)\right] \\
& \times\left(\frac{\pi^{2}}{4}-\frac{5}{3} \zeta(3)+\frac{1}{8} \int_{0}^{\infty} d x x^{3}\left(1+n_{B}(x)\right) \operatorname{Li}(x / 2)\right) .
\end{aligned}
$$

We now turn to the longitudinal case. The imaginary part of the polarization tensor for heavy, non-relativistic ions is,

$$
\operatorname{Im} \Pi_{\mathrm{L}}(Q)=\sqrt{\frac{3 \pi}{2}} \frac{k_{D}^{2}}{v_{*}} \frac{T}{q} n_{B}^{-1}\left(q_{0}\right) e^{-\frac{q_{0}^{2}}{q^{2}} \frac{3}{2 v^{* 2}}} .
$$

Its structure is such that it allows us to approximate $q_{0} \ll q$, as in the transverse case. Then, $\operatorname{Re} \Pi_{\mathrm{L}} \simeq k_{D}^{2}$, where $k_{D}$ is the Debye mass. Moreover, $\operatorname{Im}_{\mathrm{L}}$ is negligible compared with $\left(Q^{2}-\operatorname{Re}_{\mathrm{L}}(Q)\right)^{2}$ in the denominator of the spectral density, and the spectral density can be written as

$$
\beta_{\mathrm{L}}(Q) \simeq-\frac{1}{\pi} \frac{\operatorname{Im} \Pi_{\mathrm{L}}(Q)}{\left(q^{2}+k_{D}^{2}\right)^{2}}
$$


The calculation of the emission rate and energy loss rate, although more involved, proceeds in the same manner as in the transverse case. The final result is

$$
\begin{aligned}
\Gamma_{\mathrm{L}}(K)= & \frac{g_{a e}^{2}}{32 \pi^{5}} \sqrt{\frac{3 \pi}{2}} \frac{k_{D}^{2} T}{k p_{F}^{2} v_{*}}\left[2\left(\frac{p_{F}^{2}}{m_{e}^{2}}+1\right)-\frac{\mu}{p_{F}} \ln \frac{\mu+p_{F}}{\mu-p_{F}}\right] \\
& \times \int_{k}^{\infty} d q_{0}\left(1+n_{B}\left(q_{0}-k_{0}\right)\right)\left(q_{0}-k_{0}\right) e^{-\frac{q_{0}}{2 T}} \int_{q_{0}}^{\infty} \frac{q^{2} d q}{\left(q^{2}+k_{D}^{2}\right)^{2}} e^{-\frac{q_{0}^{2}}{q^{2}} \frac{3}{2 v_{*}^{2}}} .(32)
\end{aligned}
$$

The calculation of the energy loss rate may be easier, using the Parseval theorem between functions and their Fourier transforms:

$$
\int_{q_{0}}^{\infty} d q \frac{q^{2}}{\left(q^{2}+k_{D}^{2}\right)^{2}} e^{-\frac{q_{0}^{2}}{q^{2}} \frac{3}{2 v_{*}^{2}}} \simeq \frac{\sqrt{\pi}}{4 k_{D}} \int_{0}^{\infty} d x e^{-\sqrt{\frac{3}{2}} \frac{x q_{0}}{k_{D^{v}}}}\left(1+\sqrt{\frac{3}{2}} \frac{x q_{0}}{k_{D} v_{*}}\right) .
$$

Performing first the integration over $q_{0}$, and then that over $k$, an exact expression is obtained for the energy loss rate, with the remaining simple integration:

$$
\begin{aligned}
Q_{\mathrm{L}}= & \frac{g_{a e}^{2}}{128 \pi^{6}} \frac{k_{D}^{2} T^{5}}{p_{F}^{2}}\left[2\left(\frac{p_{F}^{2}}{m_{e}^{2}}+1\right)-\frac{\mu}{p_{F}} \ln \frac{\mu+p_{F}}{\mu-p_{F}}\right] \\
& \times \int_{0}^{\infty} d x e^{-\frac{x^{2}}{4}} r_{*} F^{-3}(x)\left[\left(\zeta(2, F(x)+1)+F^{-2}(x)\right)\left(\sqrt{\frac{3}{2}}+\frac{9}{2} x r_{*} F^{-1}(x)\right)\right. \\
& \left.+3 x r_{*}\left(\zeta(3, F(x)+1)+F^{-3}(x)\right)\right],
\end{aligned}
$$

with $F(x)=\frac{1}{2}\left(1+\sqrt{6} r_{*} x\right), r_{*}=\frac{T}{k_{D} v_{*}}$, and the generalized $\zeta$-function $\zeta(a, b)$.

The longitudinal energy loss rate is obviously much larger than the transverse one, by a factor $1 / v_{*}^{2}$. The result can be decomposed into an electronic part and a remaining integral, which contains the physics of Landau damping with the nuclei. This integral depends on a single parameter, $k_{D} v_{*} / T$, which can vary between 1 and 20 for the physical cases of interest. Numerically, we find that this integral is about 11., and is almost independent of $r_{*}$, within $6 \%$ accuracy, so that we might as well take its value for $k_{D}=0$. This seems to be in agreement with previous works, where it was found that the Debye screening is negligible [5]. The temperature dependence of the rate is therefore as $T^{4}$.

\section{Discussion}

Using thermal field theory methods, we have calculated the axion emission rate through the Primakoff mechanism. We have found that relativistic effects strongly 
suppress the rate at high densities, compared with the non-relativistic approximation. Nevertheless, the axion-photon coupling constraint, $G<10^{-10} \mathrm{GeV}^{-1}$ [5, 9], is not affected by these results. Indeed, this constraint comes from the evolution of $\mathrm{HB}$ stars, where the density is about $\rho \simeq 10^{4} \mathrm{~g} / \mathrm{cm}^{3}$, and where the electrons are clearly non-relativistic. For this type of coupling, axion emission has no effect in RG stars.

We have also calculated the axion emission through the electron-nucleus bremsstrahlung process, for the case of relativistic, ultra-degenerate electrons. Our final result is shown in Eq. (34). For the red giant case, the density is $\rho=10^{6} \mathrm{~g} / \mathrm{cm}^{3}$, so that we have

$$
\epsilon_{a}=g_{a e}^{2} 3.8 \times 10^{26}\left(\frac{T}{10^{8} \mathrm{~K}}\right)^{4} \mathrm{erg} / \mathrm{g} / \mathrm{s} .
$$

The difference between the non-relativistic result and the relativistic one is about a factor 2 in favor of the relativistic case. At $T=10^{8} \mathrm{~K}$, our result is almost the same as the one used in [21], where a numerical code was used to set the constraint on the axion coupling. Hence, our result translates into the following bound

$$
g_{a e}<1.5 \times 10^{-13}
$$

Should we use the usual limit for the extra energy loss rate that is tolerable, $\epsilon_{x}<$ $30 \mathrm{erg} / \mathrm{g} / \mathrm{s}$ [4], we would get a less restrictive bound, $g_{a e}<3 \times 10^{-13}$.

For the axion luminosity in white dwarfs and using the same parameters as in [4], we find

$$
L_{a}=g_{a e}^{2} 7 \times 10^{22} L_{\odot} \frac{M}{M_{\odot}}\left(\frac{T}{10^{7} \mathrm{~K}}\right)^{4},
$$

a result with the same temperature dependence as in previous works, but somewhat larger [5, 22]. Again, the origin of the discrepancy lies in the relativistic effects.

Requiring that the axion luminosity should not exceed the photon luminosity, we obtain the following bound

$$
g_{a e}<1.8 \times 10^{-13}
$$

which is sligthly weaker than the RG bound.

In the DFSZ model, Eq. (36) gives

$$
m_{a}<5.3 \times 10^{-3} \mathrm{eV} / \cos ^{2} \beta,
$$


which is as good as the SN1987A constraint, with a much lower uncertainty. In conclusion, we see that the evolution of red giants and white dwarfs leads to roughly the same constraint on the axion-electron coupling strength. These results can be summarized in a very conservative bound

$$
g_{a e}<2 \times 10^{-13} \text {. }
$$

We conclude on the cooling of G117-B15A. The authors of [2] have used a result 22] that apparently underestimates the axion emission rate by a factor 4 . Consequently, the value of $g_{a e}$ needed to account for the fast cooling of G117-B15A is weaker by a

factor of 2 compared than their estimate. A value as low as $g_{a e}=1.2 \times 10^{-13}$ would reconcile the models with the data. This may have no consequence either on the red giants evolution or on SN1987A.

\section{Acknowledgements}

We would like to thank G. Raffelt for many long and helpful mails about the Primakoff process. 


\section{References}

[1] J. E. Kim, Phys. Rep. 150 (1986) 1.

[2] J. Isern, M. Hernanz and E. Garcia-Berro, Astrophys. J. 392 (1992) L23.

[3] J. Engel, D. Seckel and A. C. Hayes, Phys. Rev. Lett. 65 (1990) 960.

[4] G. G. Raffelt, Phys. Rep. 198 (1990) 1, and references therein.

[5] G. G. Raffelt, Phys. Rev. D33 (1986) 897.

[6] A. G. Sitenko, "Electromagnetic Fluctuations in Plasmas" (Academic Press, NewYork, 1967); S. Ichimaru, "Basic Principles of Plasma Physics, A Statistical Approach" (Addison-Wesley, 1973).

[7] M. Fukujita, S Watamura and M. Yoshimura, Phys. Rev. D26 (1982) 1840.

[8] R. Chanda, J. F. Nieves and P. B. Pal, Phys. Rev. D37 (1988) 2714.

[9] G. G. Raffelt, Phys. Rev. D37 (1988) 1356.

[10] J. Kapusta, "Finite Temperature Field Theory" (The University Press, Cambridge, 1989).

[11] N. P. Landsman and Ch. G. van Weert, Phys. Rep. 145 (1987) 141.

[12] T. Altherr and U. Kraemmer, Astropart. Phys. 1 (1992) 133.

[13] R. D. Pisarski, Physica A158 (1989) 146.

[14] T. Altherr, E. Petitgirard and T. del Río Gaztelurrutia, Astropart. Phys. 1 (1993) 179.

[15] E. Braaten and D. Segel, Phys. Rev. D48 (1993) 1478.

[16] R. L. Kobes and G. W. Semenoff, Nucl. Phys. B260 (1985) 714 and B272 (1986) 329.

[17] H. A. Weldon, Phys. Rev. D28 (1983) 2007. 
[18] T. Altherr, Z. Phys. C47 (1990) 559 and Ann. Phys. (NY) 207 (1991) 374.

[19] E. Braaten and T. C. Yuan, Phys. Rev. Lett. 66 (1991) 2183.

[20] See Eq. (4.77) in [4]. Note that in our language, the axion emission rate is smaller by $1 / 2$ than the photon transition rate given by Raffelt (because of the degrees of polarization).

[21] D. Dearborn, D. Schramm and G. Steigman, Phys. Rev. Lett. 56 (1986) 26.

[22] M. Nakkagawa, Y. Kohyama and N. Itoh, Ap. J. 322 (1987) 291; M. Nakkagawa, T. Achidi, Y. Kohyama and N. Itoh, Ap. J. 326 (1988) 241. 


\section{Figure Captions}

Fig. 1 Feynman diagram for the Primakoff process.

Fig. 2 Axion energy loss rate due to the Primakoff effect for a wide range of densities typical of red giant stars. The solid curve is our result (Eq. (11)). Also plotted are Raffelt's result (dashed curve) and the pole-cut rate with transverse photons only (dotted curve). The value of the axion-photon coupling has been fixed to $G=10^{-10} \mathrm{GeV}^{-1}$.

Fig. 3 The two cut graphs that are needed to evaluate the bremsstrahlung contribution to the axion emission rate. 
This figure "fig1-1.png" is available in "png" format from: http://arxiv.org/ps/hep-ph/9310304v1 
This figure "fig1-2.png" is available in "png" format from: http://arxiv.org/ps/hep-ph/9310304v1 